\title{
Obesity and nonalcoholic fatty liver disease: current perspectives
}

This article was published in the following Dove Press journal: Diabetes, Metabolic Syndrome and Obesity:Targets and Therapy

\author{
Raiya Sarwar' \\ Nicholas Pierce ${ }^{2}$ \\ Sean Koppe ${ }^{3}$ \\ 'Department of Internal Medicine, \\ University of Illinois at Urbana- \\ Champaign, Champaign, IL, USA; \\ ${ }^{2}$ University of Medicine and Health \\ Sciences, New York, NY, USA; \\ ${ }^{3}$ Department of Medicine, Division \\ of Gastroenterology and Hepatology, \\ University of Illinois at Chicago, \\ Chicago, IL, USA
}

\begin{abstract}
Nonalcoholic fatty liver disease (NAFLD) is rapidly becoming the most common cause of chronic liver disease due to an increase in the prevalence of obesity. The development of NASH leads to an increase in morbidity and mortality. While the first line of treatment is lifestyle modifications, including dietary changes and increased physical activity, there are no approved pharmacological treatment agents for NAFLD and NASH currently. Due to its complex pathophysiology, different pathways are under investigation for drug development with the focus on metabolic pathways, inflammation, and slowing or reversing fibrosis. There are several agents advancing in clinical trials, and promising results have been seen with drugs that affect hepatic steatosis, inflammation, and fibrosis. This review will provide an overview on NAFLD and some of the mechanisms of disease that are being targeted with pharmacologic agents.
\end{abstract}

Keywords: nonalcoholic fatty liver disease, nonalcoholic steatohepatitis, pharmacotherapy, clinical trials, antifibrotics

\section{Introduction}

Nonalcoholic fatty liver disease (NAFLD) has become the most common cause of chronic liver disease in the United States. NAFLD is associated with metabolic disorders, such as type 2 diabetes mellitus, hypertension, dyslipidemia, and obesity. The prevalence of NAFLD is estimated to be between $20 \%$ and $30 \%$ in the USA, and in the upcoming decade, it is also expected to be the leading cause of liver transplantation. ${ }^{1,2}$ NAFLD exists on a spectrum from simple steatosis to steatohepatitis (nonalcoholic steatohepatitis [NASH]), which is marked by lobular inflammation and ballooning. Across the spectrum of disease, fibrosis progression can lead to the development of cirrhosis, although fibrosis progression is typically more common and rapid with NASH as opposed to simple steatosis. It is important to note that not all patients with NASH will progress to cirrhosis and understanding which patients will progress and require treatment is an area of active investigation. Additionally, there are multiple potential therapeutic targets that are being explored for NASH and multiple novel therapies are on the horizon. In this article, the pathways and mechanisms of potential drugs and treatments will be reviewed.

\section{Background}

The pathophysiology of NASH is complex, multifactorial, and still under investigation. It is generally accepted that multiple pathways and mechanisms are involved in the 
pathogenesis of NASH. These mechanisms include dietary factors, insulin resistance, genetic polymorphisms, lipotoxicity, and altered gut microbiota. NAFLD can be diagnosed with the evidence of hepatic steatosis on imaging or histology and lack of secondary causes of hepatic fat accumulation (alcoholic steatosis, medications, or hereditary disorders). Nonalcoholic fatty liver (NAFL) is defined as the presence of $\geq 5 \%$ hepatic steatosis without evidence of hepatocellular injury in the form of hepatocyte ballooning. While the majority of patients tend to remain in the benign NAFL stage, some patients progress to NASH, which is characterized by the presence of $\geq 5 \%$ steatosis and inflammation with hepatocyte injury, with or without fibrosis. ${ }^{3}$ An established scoring system for the assessment of histology in NAFLD is the NAFLD activity score (NAS). The NAS is quantified using the following characteristics: steatosis (0-3), lobular inflammation (0-3), and hepatocyte ballooning (0-2), which are added together to arrive at a final score $(0-8) .{ }^{4}$ NAS has now become frequently used as an inclusion criteria for clinical trials, and improvement in the NAS is often used as an endpoint as well. Ultimately, the liver histology can also exhibit signs of fibrosis, which raises concern for progressive and more advanced liver disease. NASH reflects hepatocellular damage and often the commencement of fibrosis progression, and yet, several long-term outcome studies have suggested that it is the fibrosis stage rather than the presence of NASH or an elevated NAS that predicts patient outcomes. ${ }^{5,6}$ This may be a reflection of retrospective studies with insufficient power or it may be that NASH is a more dynamic entity, which may spontaneously resolve as opposed to fibrosis, the presence of which is more intractable. The most valued endpoints for current clinical trials have thus become the "resolution of NASH" and/or the "improvement of fibrosis."

\section{Lifestyle modifications}

Lifestyle modification is the first line of treatment for patients with NAFLD, which includes diet modification, sustained weight loss, and increase in exercise. In a meta-analysis, it was shown that $5 \%$ body weight reduction resulted in improvement in hepatic steatosis, whereas $>7 \%$ reduction was associated with NAS improvement. ${ }^{7}$ These data have been supported by a prospective trial in which lifestyle modification to induce weight loss was started in 261 subjects with liver biopsies before and after the intervention. High rates of NASH resolution and fibrosis regression were noted in patients with $\geq 10 \%$ weight loss. $^{8}$ Calorie-restricted diet is associated with mobilization of liver fat, which also reduces cardiovascular $(\mathrm{CV})$ risk. Mediterranean diet reduces the risk of $\mathrm{CV}$ diseases and has also been shown to improve steatosis. ${ }^{9}$ Mediterranean diet has been studied along with low-fat, high-carbohydrate diet in a 6-week trial in patients with biopsy-proven NAFLD and showed reduction in hepatic steatosis and improved insulin sensitivity. ${ }^{10}$ A recent study that included 58 patients with NAFLD matched with 58 controls concluded that adherence to a Mediterranean diet is associated with less severe liver disease in patients with NAFLD. ${ }^{11}$ Increased protein intake may also be beneficial as a prospective study with 37 subjects with type 2 diabetes, placed on isocaloric, high-protein diet for 6 weeks, which showed reduced liver fat by $36 \%-48 \%$ and a decrease in serum fibroblast growth factor-21 (FGF21) correlating with hepatic fat loss. ${ }^{12}$ Increase in exercise and physical activity is also beneficial for NAFLD. Data suggest an improvement in hepatic steatosis with exercise in patients involved in sustained physical activity more than 150 minutes per week and patients who increase their physical activity more than 60 minutes per week have shown a decrease in serum aminotransferases. ${ }^{13}$ Increased exercise in combination with diet modification is often recommended simultaneously in patients with NAFLD.

\section{Current treatment options} Vitamin $\mathrm{E}$ and pioglitazone

There are currently no Food and Drug Administration (FDA)-approved medications to treat NASH. Despite not being FDA-approved specifically for NASH, treating NASH with vitamin $\mathrm{E}$ or pioglitazone is a consideration for certain patients. Vitamin $\mathrm{E}$ is an antioxidant that works as a free radical scavenger and protects cellular components from peroxidation. The PIVENS trial (Pioglitazone vs Vitamin E vs Placebo for Treatment of Non-Diabetic Patients With Nonalcoholic Steatohepatitis) studied the effects of both pioglitazone and vitamin $\mathrm{E}$ in nondiabetic patients with biopsy-proven NASH. Two hundred forty-seven patients were randomized to pioglitazone, vitamin E, or placebo for 24 months. Inclusion criteria were definite or possible steatohepatitis with an NAS of 5 or more or definite steatohepatitis with an NAS of 4. Exclusion criteria included alcohol consumption, presence of cirrhosis, hepatitis $\mathrm{C}$, heart failure, or use of medications known to cause steatohepatitis. Primary outcome was improvement in histological features of NASH. Results from PIVENS trial showed that vitamin E reduced the serum aminotransferase levels and was associated with a significantly higher rate of improvement in nonalcoholic steatohepatitis (43\% vs 19\%, $P=0.001) .{ }^{14}$ In the TONIC (Treatment of Nonalcoholic Fatty Liver Disease in Children) trial, vitamin $\mathrm{E}$ was evaluated 
along with metformin or placebo in children with biopsyproven NAFLD, and there was a significant increase in NASH resolution in children treated with vitamin $\mathrm{E}$ compared with placebo. ${ }^{15}$ According to the 2018 practice guidelines recommended by American Association of Study of Liver Diseases, vitamin E (RRR- $\alpha$-tocopherol) administered at a dose of 800 IU/day should be considered for nondiabetic patients with biopsy-proven NASH. ${ }^{3}$ While vitamin E appears to have benefit in the treatment of NASH, debate about its influence on all-cause mortality and its influence on prostate cancer tempers enthusiasm for its use. ${ }^{16-18}$

Thiazolidinediones are peroxisome proliferator-activated receptor gamma (PPAR- $\gamma$ ) ligands and have been studied in NASH due to their ability to reverse adipose tissue dysfunction and insulin resistance. In the PIVENS trial mentioned above, comparison of pioglitazone therapy with placebo did not reach the prespecified $P$-value of 0.025 for the primary outcome ( $34 \%$ vs $19 \%, P=0.04$; number needed to treat 6.9) but achieved the secondary endpoint of NASH resolution. ${ }^{14}$ Weight gain was the most common side effect with pioglitazone. An additional meta-analysis suggests a benefit of pioglitazone in diabetic and nondiabetic patients with NASH. ${ }^{19}$ Excitement for pioglitazone is affected by concerns about weight gain, potential ill effects on bone density in certain populations, and a questionable influence on bladder cancer. ${ }^{19,20}$

\section{Use of metformin in NASH}

Metformin has shown benefits regarding improvement in insulin resistance and weight loss in diabetic patients. Several studies have shown it to be effective for improvement in serum aminotransferases but two meta-analysis showed that metformin therapy did not improve liver histology. ${ }^{21,22}$

\section{Use of statins in NAFLD and NASH}

Patients with NAFLD often have elevated serum triglyceride (TG), low-density lipoprotein (LDL), and high apolipoprotein $\beta$ to apolipoprotein A-1 ratio, with low high-density lipoprotein (HDL) levels. ${ }^{23}$ These changes are driven by hepatic lipid concentration and insulin resistance. Treatment of dyslipidemia reduces the risk of $\mathrm{CV}$ disease. The GREACE study (The GREek Atorvastatin and Coronary-heart-disease Evaluation) showed improvement in aminotransferases and CV outcomes with statins in patients with NAFLD-related elevated aminotransferases. ${ }^{24}$ The IDEAL trial (Initiating Dialysis Early and Late) suggested a benefit of high-intensity statins compared with moderate-intensity statins in patients with baseline elevated alanine aminotransferase (ALT). ${ }^{25}$
Statin-induced hepatotoxicity is not higher in patients with chronic liver disease, and pravastatin has been specifically studied in a prospective randomized controlled trial with 326 subjects with NASH and chronic hepatitis C. Pravastatin significantly lowered LDL cholesterol, total cholesterol (TC), and TG values at 12 weeks in comparison with placebo and was found to be safe and efficacious. The study demonstrated that hypercholesterolemic patients with compensated chronic liver disease can be treated with statins. ${ }^{26}$ Statin use has also been shown to be inversely related to the risk of death or liver transplant in patients with NAFLD. ${ }^{5}$ Despite the safety of statins in patients with NAFLD and their apparent benefit, statins are actually often underprescribed in patients with NAFLD. $^{27}$

\section{NASH clinical trials and their endpoints}

A treatment agent can have different impacts on the NASH progression. Pharmacological agents can improve NASH or may influence fibrosis. These parameters need to be evaluated simultaneously to assess the potential benefits of any agent and to be sure that improvement in one does not worsen the other. The endpoint determination in NASH is challenging due to a long natural history of disease, which in turn raises the need to determine appropriate short-term surrogate markers for accelerated drug development pathway.

\section{Histologic endpoints}

Histologic endpoints to assess response to therapeutic intervention can be defined as improvement in NAS, resolution of NASH, or improvement in liver fibrosis. ${ }^{28}$ When NAS is used as primary outcome, it is recommended that two-point improvement in the total score should be achieved with no worsening of fibrosis. For resolution of NASH, it is defined as complete resolution of hepatocyte ballooning with inflammation score of 0 or 1 and no worsening of fibrosis. Hepatocyte ballooning is an important parameter in NASH and has been shown to correlate with progressive disease and fibrosis. The fat accumulation in the ballooned hepatocyte causes oxidative injury, endoplasmic reticulum dysfunction, and abnormalities of cytoskeleton evident as Mallory-Denk body. ${ }^{29}$ Therefore, it is an important endpoint used in many of the drug trials.

For the trials focusing on the assessment of fibrosis, there should be no worsening of fibrosis in NASH or ideally an intervention showing some improvement of fibrosis.

\section{Biomarkers as surrogates}

Noninvasive modalities are gaining interest in the evaluation of steatosis, inflammation, and fibrosis. For hepatic steatosis 
assessment, controlled attenuation parameter as part of vibration-controlled transient elastography (FibroScan) and MRI proton density fat fraction (MRI-PDFF) and multiparametric MRI (LiverMultiScan) are being used. For liver inflammation and ballooning, liver enzymes ALT and aspartate aminotransferase (AST) can be used. LiverMultiScan has gained attention for inflammation assessment but requires confirmatory data. ${ }^{30}$ Transient elastography, LiverMultiScan, MRI, or MR elastography can be used for analysis of fibrosis. ${ }^{31-33}$ There are limited biomarkers available for accurate hepatocyte fibrosis assessment at this time, but Pro-C3, FIB-4, NAFLD fibrosis score, and the enhanced liver fibrosis score are commonly investigated noninvasive tools for fibrosis assessment. ${ }^{3}$

\section{Clinical endpoints}

Clinical endpoints depend on the stage being studied. In earlystage disease, the outcome assessment is challenging due to dynamic and generally indolent nature of disease progression. In the setting of cirrhosis, the endpoints might include changes in the degree of portal hypertension or hepatic decompensation. Changes in portal hypertension can be assessed by measurement in the hepatic venous pressure gradient. Hepatic decompensation can be evaluated by Child-Turcotte-Pugh score, model for end-stage liver disease score, and development of new or worsening in ascites, variceal bleed, hepatic encephalopathy, and hepatocellular carcinoma (HCC). Methacetin breath test and HepQuant are under investigation and can be used to assess changes in liver function.

\section{Pharmacological agents with completed phase II and undergoing phase III}

Several pharmacological agents investigated for the treatment of NASH. Therapies are at various stages of drug development (Table 1 and Figure 1).

\section{Obeticholic acid}

Obeticholic acid (OCA) is derived from chenodeoxycholic acid, which is a natural ligand for farnesoid $\mathrm{X}$ receptor (FXR). ${ }^{34}$ It regulates bile acid synthesis and transport, increases glucose-stimulated insulin secretion and peripheral glucose uptake, inhibits hepatic lipid synthesis by inhibiting CYP7A1 thus acting on the rate-limiting step for conversion of cholesterol to bile acids, and increases lipid uptake by adipocytes. ${ }^{34}$ It may also decrease the portal pressure and possess anti-inflammatory and antifibrotic activity. ${ }^{28,35,36}$ OCA was studied in the FLINT (Farnesoid X nuclear receptor ligand OCA for noncirrhotic, nonalcoholic steatohepatitis treatment) trial, which was a double-blind, randomized, placebo-controlled, phase IIb trial in patients with biopsy-proven NASH (NAS $\geq 4$ ) without cirrhosis. ${ }^{37}$ Patients were randomized to OCA $25 \mathrm{mg}$ daily or placebo for 72 weeks. Patients in the treatment arm had significant histologic improvement with two-point improvement in NAS without worsening in fibrosis $(P=0.0002)$. Interestingly, patients treated with OCA showed improvement in fibrosis (35\% vs 19\%), hepatocyte ballooning (46\% vs 31\%), steatosis (61\% vs 38\%), and lobular inflammation (53\% vs $35 \%$ ) compared with placebo. It was also associated with significant reduction in ALT, AST, $\gamma$-glutamyltransferase, and bilirubin. However, there was a modest increase in TC and LDL with a corresponding decrease in HDL raising some concern, given the increased CV risk that patients with NASH already possess. These changes did not sustain after completion of study or could generally be attenuated with the use of statins. ${ }^{38}$ Pruritus was also noted as adverse effect in $23 \%$ of subjects on OCA treatment with severe pruritus leading to stopping OCA in one subject. OCA is being evaluated in the REGENERATE trial, which is a phase III trial with a target of 2,000 subjects with biopsy-proven NASH and stage 1-3 fibrosis randomized to $10 \mathrm{mg}, 25 \mathrm{mg}$ OCA, or placebo for 72 weeks. Primary endpoints are reduction in fibrosis one point without worsening of NASH, resolution of NASH, all-cause mortality, and liver-related clinical outcomes. The initial histopathological analysis will be at 18 months with subsequent analysis at 48 months followed by final assessment of outcomes at 6 years. ${ }^{28,39}$

\section{Elafibranor}

Elafibranor (GFT-505) is a dual peroxisome proliferatoractivated receptor alpha-delta (PPAR- $\alpha / \delta)$ agonist, which is currently in phase III. PPAR- $\alpha$ receptor plays an important role by controlling lipid flux by modulation of fatty acid transport and oxidation and decreases TGs and increases HDL. ${ }^{34}$ It also inhibits inflammatory genes and decreases acute-phase response gene expression. PPAR- $\delta$ improves glucose homeostasis, fatty acid transport, and oxidation and has an anti-inflammatory effect on Kupffer cells. It is also highly expressed in the adipocytes controlling adipocyte differentiation, promoting fatty acid uptake, and increasing insulin sensitivity. ${ }^{28,40,41}$ GOLDEN-505 trial was a double-blinded, placebo-controlled, randomized, international phase IIb trial with biopsy-proven NASH without cirrhosis in 276 subjects. ${ }^{42}$ The primary outcome was resolution of NASH defined as disappearance of steatosis, ballooning, or lobular inflammation. Subjects were randomized to receive elafibranor $80 \mathrm{mg}$ vs $120 \mathrm{mg}$ vs placebo for 52 weeks. The primary outcome was 
Table I Pharmacological therapies for NASH

\begin{tabular}{|c|c|c|c|}
\hline $\begin{array}{l}\text { Agent and } \\
\text { mechanism }\end{array}$ & Effect & Phase & Key inclusion and exclusion criteria \\
\hline $\begin{array}{l}\text { Obeticholic acid } \\
\text { (OCA) } \\
\text { FXR receptor } \\
\text { agonist }\end{array}$ & $\begin{array}{l}\text { Improvement in NAS, steatosis, } \\
\text { ballooning, and fibrosis }\end{array}$ & $\begin{array}{l}\text { REGENERATE, phase III trial. Primary } \\
\text { endpoint reduction in fibrosis } \geq I \text { point } \\
\text { without worsening of NASH, resolution } \\
\text { of NASH, all-cause mortality, and liver- } \\
\text { related clinical outcomes }\end{array}$ & $\begin{array}{l}\text { - Stage I-3 fibrosis } \\
\text { - No cirrhosis } \\
\text { - No BMI >45 } \\
\text { - No significant alcohol or other liver disease } \\
\text { - No poorly controlled diabetes }\end{array}$ \\
\hline $\begin{array}{l}\text { Elafibranor } \\
\text { PPAR } \alpha / \delta \text { agonist }\end{array}$ & $\begin{array}{l}\text { Modulation of fatty acid } \\
\text { transport and } \beta \text {-oxidation, } \\
\text { improves glucose homeostasis, } \\
\text { and has anti-inflammatory effect }\end{array}$ & $\begin{array}{l}\text { RESOLVE-IT, phase III, randomized, } \\
\text { placebo-controlled trial } \\
\text { Primary endpoint NASH resolution } \\
\text { without worsening fibrosis }\end{array}$ & $\begin{array}{l}\text { - Stage I-3 fibrosis } \\
\text { - No cirrhosis } \\
\text { - No poorly controlled diabetes } \\
\text { - No significant alcohol use }\end{array}$ \\
\hline $\begin{array}{l}\text { Cenicriviroc } \\
\text { CCR2/CCR5 } \\
\text { receptor antagonist }\end{array}$ & $\begin{array}{l}\text { Anti-inflammatory and } \\
\text { antifibrotic activity }\end{array}$ & $\begin{array}{l}\text { AURORA, randomized, double-blind, } \\
\text { placebo controlled trial. Primary } \\
\text { endpoint improvement in fibrosis } \\
\text { without worsening of steatohepatitis }\end{array}$ & $\begin{array}{l}\text { - Stage I-3 fibrosis } \\
\text { - No cirrhosis } \\
\text { - No significant alcohol use or other liver } \\
\text { disease }\end{array}$ \\
\hline $\begin{array}{l}\text { Selonsertib } \\
\text { ASK-I inhibitor }\end{array}$ & Inhibits inflammation and fibrosis & $\begin{array}{l}\text { STELLAR } 3 \text { - phase III trial in patients } \\
\text { with bridging fibrosis; STELLAR } 4 \text { - } \\
\text { patients with compensated cirrhosis. } \\
\text { Primary endpoint improvement in } \\
\text { fibrosis without worsening NASH }\end{array}$ & $\begin{array}{l}\text { - STELLAR } 3 \text { - stage } 3 \text { fibrosis } \\
\text { - STELLAR } 4 \text { - stage } 4 \text { fibrosis } \\
\text { - No decompensated cirrhosis }\end{array}$ \\
\hline $\begin{array}{l}\text { Aramchol } \\
\text { Synthetic lipid } \\
\text { molecule }\end{array}$ & $\begin{array}{l}\text { Decreases lipogenesis and } \\
\text { increases } \beta \text {-oxidation }\end{array}$ & $\begin{array}{l}\text { Phase Ilb trial. Primary endpoint liver } \\
\text { triglyceride change }\end{array}$ & $\begin{array}{l}\text { - Stage I-3 fibrosis } \\
\text { - No cirrhosis }\end{array}$ \\
\hline $\begin{array}{l}\text { Emricasan } \\
\text { Pancaspase inhibitor }\end{array}$ & $\begin{array}{l}\text { Decreases portal hypertension } \\
\text { and blocks apoptotic and } \\
\text { inflammatory caspase activation }\end{array}$ & $\begin{array}{l}\text { ENCORE-NF, phase llb trial. Primary } \\
\text { endpoint improvement in fibrosis } \\
\text { without worsening of NASH }\end{array}$ & $\begin{array}{l}\text { - Stage I-3 fibrosis } \\
\text { - No cirrhosis }\end{array}$ \\
\hline $\begin{array}{l}\text { NGM282 } \\
\text { Recombinant FGFI9 } \\
\text { agonist }\end{array}$ & $\begin{array}{l}\text { Inhibits bile acid formation and } \\
\text { improves insulin sensitization }\end{array}$ & $\begin{array}{l}\text { Phase lla trial. Primary endpoint change } \\
\text { in hepatic steatosis }\end{array}$ & $\begin{array}{l}\text { - Any diagnosis of NASH } \\
\text { - No clinically significant acute or chronic } \\
\text { liver disease }\end{array}$ \\
\hline $\begin{array}{l}\text { BMS-986036 } \\
\text { Recombinant FGF2I } \\
\text { agonist }\end{array}$ & $\begin{array}{l}\text { Decreases hepatic } \\
\text { gluconeogenesis and lipogenesis }\end{array}$ & $\begin{array}{l}\text { Phase lla trial. Primary endpoint change } \\
\text { in hepatic fat fraction }\end{array}$ & $\begin{array}{l}\text { - Any diagnosis of NASH } \\
\text { - No evidence for alternative liver diseases }\end{array}$ \\
\hline $\begin{array}{l}\text { GS- } 0976 \\
\text { Acetyl-CoA } \\
\text { carboxylase inhibitor }\end{array}$ & Downregulation of steatosis & $\begin{array}{l}\text { Phase Ila trial. Primary outcome safety } \\
\text { evaluation }\end{array}$ & $\begin{array}{l}\text { - } \text { MRI-PDFF }>8 \% \text { steatosis } \\
\text { - Stage I-3 fibrosis } \\
\text { - } \text { MR elastography } \geq 2.5 \mathrm{kPa} \\
\text { - No cirrhosis } \\
\text { - No alcohol use }\end{array}$ \\
\hline $\begin{array}{l}\text { MGL-3196 } \\
\text { Thyroid hormone } \\
\text { receptor } \beta \text {-agonist }\end{array}$ & $\begin{array}{l}\text { Decreases TGL, LDL, and } \\
\text { PCSK-9 and increases reverse } \\
\text { cholesterol metabolism }\end{array}$ & $\begin{array}{l}\text { Phase lla trial. Primary endpoint change } \\
\text { in hepatic fat content }\end{array}$ & $\begin{array}{l}\text { - } \text { MRI-PDFF }>10 \% \text { steatosis } \\
\text { - Stage I-3 fibrosis } \\
\text { - No cirrhosis }\end{array}$ \\
\hline $\begin{array}{l}\text { Liraglutide } \\
\text { GLP-I analog }\end{array}$ & $\begin{array}{l}\text { Increases free fatty acid } \\
\beta \text {-oxidation }\end{array}$ & $\begin{array}{l}\text { LEAN trial phase lla. Primary endpoint } \\
\text { resolution of NASH without worsening } \\
\text { fibrosis }\end{array}$ & $\begin{array}{l}\text { - No poorly controlled diabetes } \\
\text { - No advanced cirrhosis }\end{array}$ \\
\hline
\end{tabular}

Abbreviations: NASH, nonalcoholic steatohepatitis; FXR, farnesoid $X$ receptor; PPAR, peroxisome proliferator-activated receptor; CCR2/CCR5, chemokine receptor 2/chemokine receptor 5; ASK-I, apoptosis signal-regulating kinase I; FGF, fibroblast growth factor; GLP-I, glucagon-like peptide-I; NAS, NAFLD activity score; NAFLD, nonalcoholic fatty liver disease; LDL, low-density lipoprotein; BMI, body mass index; PDFF, proton density fat fraction; TGL, triglycerides.

not achieved as there was no statistically significant difference in three groups. However, the subjects with NASH and NAS $\geq 4$ showed higher proportion of NASH resolution compared with placebo in the elafibranor $120 \mathrm{mg}$ group ( $20 \%$ vs $11 \%$, $P=0.018$ ) along with significant histologic improvement in fibrosis in correlation with changes in ballooning and lobular inflammation. ${ }^{42}$ Elafibranor also resulted in favorable effects on the lipid profile with decreased TC, LDL, and TGs, while increasing HDL. Elafibranor also improved glucose homeostasis in diabetic subjects in the $120 \mathrm{mg}$ arm. There was no weight gain as well, but a mild reversible elevation in serum creatinine was noted. RESOLVE-IT is a randomized, placebocontrolled phase III trial investigating these findings in 2,000 subjects with biopsy-proven NASH (NAS $\geq 4$ ) and fibrosis stage 1-3. Subjects will be randomized to elafibranor 120 $\mathrm{mg}$ or placebo. The primary endpoint of NASH resolution 


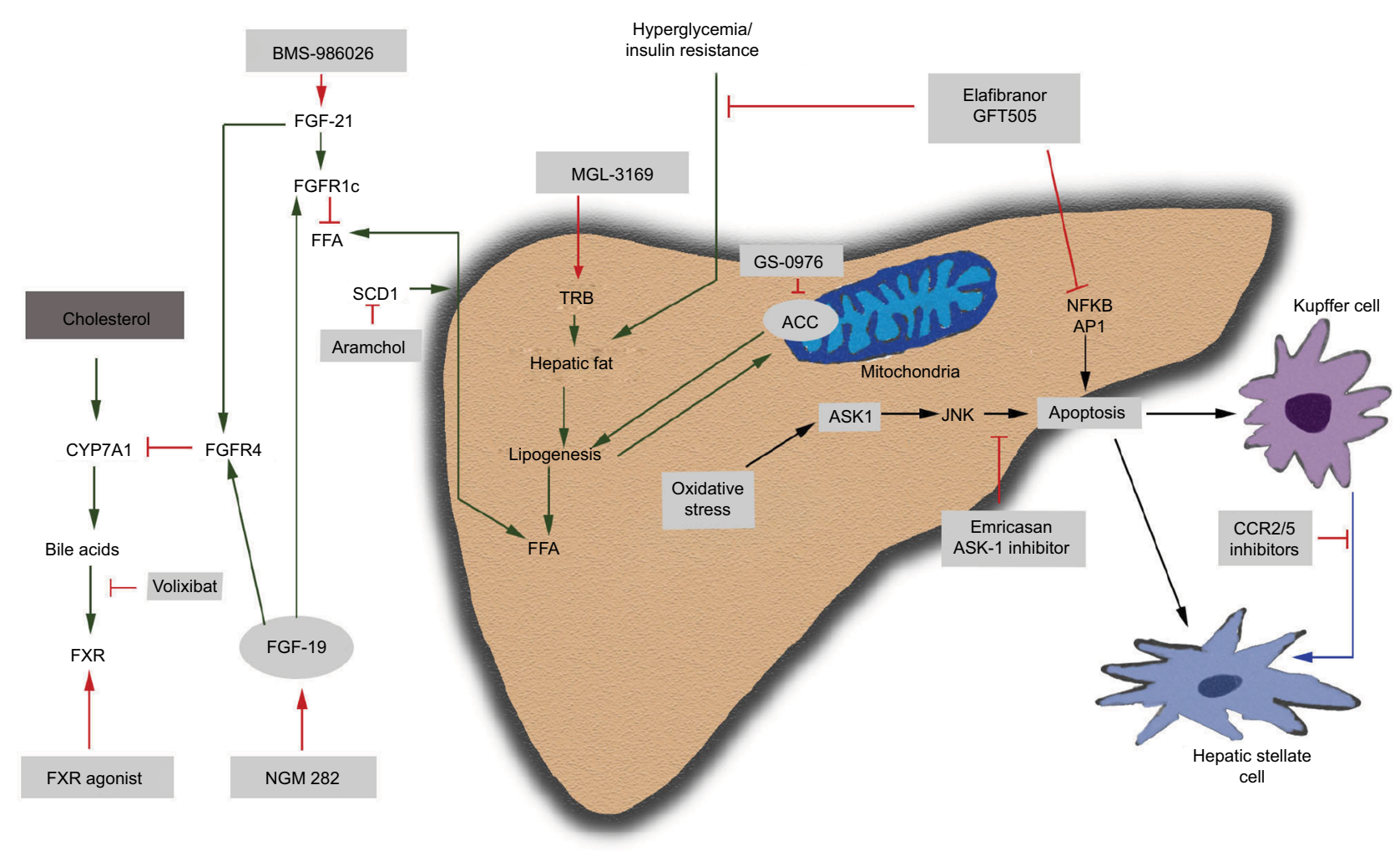

Figure I Mechanism of action of pharmacotherapies for NASH.

Abbreviations: ACC, acetyl-CoA carboxylase; API, activator protein I; ASK, apoptosis signal-regulating kinase; CCR2/5, C-C chemokine receptor type 2/5; CYP7AI, cytochrome P450 7AI; ER, endoplasmic reticulum; FFA, free fatty acids; FGF, fibroblast growth factor; FGFR, fibroblast growth factor receptor; FXR, Farnesoid X receptor; JNK, Jun N-terminal kinases; NASH, nonalcoholic steatohepatitis; ROS, reactive oxygen species; SCD, stearoyl-CoA desaturase; TR $\beta$, thyroid receptor $\beta$.

without worsening fibrosis will be analyzed at 72 weeks. The study is estimated to last 4 years until the achievement of prespecified number of events of interest, all-cause mortality, and liver-related clinical outcomes.

\section{Cenicriviroc}

Cenicriviroc (CVC) is a CCR2/CCR5 chemokine receptor antagonist, which inhibits macrophage recruitment and migration to the liver resulting in reduced inflammation and an antifibrotic effect. ${ }^{43,44} \mathrm{CVC}$ was evaluated in the CENTAUR study (Efficacy and Safety study of Cenicriviroc for Treatment of NASH in Adult Subjects With Liver Fibrosis), a phase IIb, randomized, placebo-controlled study of 289 subjects with biopsy-proven NASH (NAS $\geq 4$, fibrosis stage 1-3) and diabetes or the metabolic syndrome. Subjects were randomized to CVC $150 \mathrm{mg}$ daily for 2 years vs placebo for 1 year followed by CVC $150 \mathrm{mg}$ daily for the next 1 year. At the end of the first year, the analysis did not show statistically significant rate of endpoint achievement; however, $20 \%$ of subjects in CVC arm achieved improvement in fibrosis by one stage without worsening steatohepatitis. ${ }^{28,38,45}$ A phase III clinical trial will assess the impact on fibrosis in subjects with NASH and fibrosis stage 2-3.

\section{Selonsertib}

Apoptosis signal-regulating kinase 1 (ASK-1) is an important pathway that can result in apoptosis and fibrosis. ASK-1 is activated by oxidative stress and inflammatory markers, which results in the production of inflammatory cytokines, expression of matrix remodeling genes, and promoting apoptotic cell death. ${ }^{34}$ Selonsertib (SEL) is an ASK-1 inhibitor studied in a phase IIb trial of 72 patients with biopsy-proven NASH, with NAS $\geq 5$ and fibrosis stage 2-3. SEL was studied in combination with simtuzumab (SIM), which is an injectable monoclonal antibody to lysyl oxidase-like $2 .{ }^{38}$ SIM was initially believed to have an antifibrotic effect although its development was stopped for lack of efficacy, and in this particular trial, it is now viewed in hindsight as no better than placebo. Subjects were randomized 2:2:1:1:1 to receive SEL $18 \mathrm{mg}$ or $6 \mathrm{mg} \pm$ SIM weekly vs SIM alone for 24 weeks. Fibrosis improvement was noted in $43 \%$ of subjects in SEL $18 \mathrm{mg}+$ SIM in comparison with $30 \%$ in SEL $6 \mathrm{mg}+$ SIM and $20 \%$ in SIM alone arm (ie, placebo). ${ }^{28,46}$ There are currently two phase III trials for evaluation of safety and efficacy of SEL vs placebo in adults with bridging fibrosis (STELLAR 3) and compensated cirrhosis (STELLAR 4). The primary endpoint in both the trials is improvement in fibrosis without worsening of NASH. ${ }^{28,47,48}$ 


\section{Promising pharmacological agents in phase IIA and IIB trials}

NGM282 is a recombinant human fibroblast growth factor FGF19 agonist that binds to FGFR4 and FGFR1c. It not only inhibits bile acid formation from cholesterol by blocking CYP7a1 enzyme but also improves insulin sensitization and is believed to have favorable metabolic effects. ${ }^{49} \mathrm{It}$ has been studied in a recent phase IIa, randomized, placebo-controlled trial by enrolling 82 patients with biopsy-confirmed NASH (NAS $\geq 4$ with one point in each component, fibrosis stage $1-3)$, with $8 \%$ liver fat content by MRI-PDFF and abnormal ALT. Subjects were randomized to $3 \mathrm{mg}, 6 \mathrm{mg}$ of NGM282, or placebo as a daily subcutaneous injection for 12 weeks. The primary endpoint was focused on assessing the change in hepatic steatosis $(5 \%$ reduction in absolute liver fat content by MRI-PDFF). There was a significant reduction in liver fat content noted in the treatment arm. There was a significant absolute reduction in liver fat in both arms, with a $9.7 \%$ reduction of liver fat in the $3 \mathrm{mg}$ arm and an $11.9 \%$ absolute reduction in liver fat in the $6 \mathrm{mg}$ arm. ALT normalization was also achieved in $35 \%$ and $37 \%$ of subjects with $3 \mathrm{mg}$ and $6 \mathrm{mg}$, respectively, at 12 weeks. NGM282 also resulted in reduced serum markers of fibrosis; however, a concern is a significant increase in LDL $(>30 \mathrm{mg} / \mathrm{dL}$ increase in both treatment arms). The common side effects were increased stool frequency, loose stool, nausea, and injection site erythema. ${ }^{28,50}$

BMS-986036 is a recombinant FGF21 agonist, which mainly acts via FGFR1c pathway but also acts through FGFR2 and FGFR3. It improves glycemic control by decreasing hepatic gluconeogenesis, improves insulin sensitivity, decreases lipogenesis, and has a possible weight loss effect. A phase IIa, randomized, double-blind, placebo-control study enrolled 74 patients with biopsyproven NASH fibrosis stage 1-3, BMI25, and hepatic fat fraction $10 \%$ on MRI-PDFF. Patients received BMS$98603610 \mathrm{mg}$ daily (QD), BMS-986036 $20 \mathrm{mg}$ weekly (QW), or placebo daily for 16 weeks. Primary endpoint was change in hepatic fat fraction by MRI-PDFF. Both treatment arms had a significant reduction in hepatic fat content, which was analyzed as absolute change in MRIPDFF (-6.8\% in $10 \mathrm{mg}$ daily, $-5.2 \%$ in $20 \mathrm{mg}$ weekly, vs $-1.3 \%$ in placebo arm). There was also an improvement in ALT, pro-C3 (serum marker of fibrosis), and magnetic resonance elastography (MRE). ${ }^{51}$

Glucagon-like peptide-1 (GLP-1) is a hormone secreted by $\mathrm{L}$ cells in the distal ileum and regulates the insulin secretion after food intake and can also decrease gastric emptying and result in reduced appetite. It also causes an increase in PPAR- $\alpha$ and PPAR- $\gamma$ expression, resulting in free fatty acid $\beta$-oxidation. ${ }^{34}$ GLP-1 liraglutide (LEADER trial) and semaglutide (SUSTAIN-6 trial) have been shown to reduce $\mathrm{CV}$ risk in type 2 diabetic patients with increased CV risk. ${ }^{52}$ The LEAN trial (Liraglutide efficacy and action in nonalcoholic steatohepatitis), a phase II, randomized, multicenter, double-blind, placebo-controlled trial, evaluated 52 patients with liraglutide $1.8 \mathrm{mg}$ daily (subcutaneous injections) vs placebo for 48 weeks. The primary outcome was resolution of definite NASH with no worsening in fibrosis. There was resolution of NASH in $39 \%$ of subjects receiving liraglutide vs $9 \%$ of subjects in the placebo group $(P=0.019) .{ }^{53}$ Liraglutide use also resulted in weight loss, and further investigation into the potential of GLP-1 agents for NASH is warranted.

Acetyl-CoA carboxylase (ACC) inhibition reduces lipogenesis and increases lipid oxidation. GS-0976 is an ACC inhibitor and inhibits the conversion of acetyl-CoA to malonyl-CoA and downregulates steatosis. ${ }^{34}$ Preliminary data from a phase IIa clinical trial over a 12-week period involving 10 subjects with NASH receiving $20 \mathrm{mg}$ of GS-0976 daily showed significant improvement in liver fat content by MRI-PDFF and noninvasive markers of fibrosis, as well as improvement in liver stiffness assessed by MRE. ${ }^{28}$ A combination regimen of GS-0976 and GS-9674 (a FXR agonist similar to OCA) is also being explored in a phase IIa clinical trial.

NAFLD is associated with a high rate of overt and subclinical hypothyroidism. It results in decreasing thyroid hormone-regulated gene expression in liver consistent with liver-specific hypothyroidism. Hypothyroidism is associated with increased TGs and cholesterol. ${ }^{54}$ Thyroid hormone receptor $\beta$-agonist decreases free fatty acids and TGs and has been shown to reduce liver steatosis as well. ${ }^{37,38,55}$ MGL-3196 is a thyroid hormone receptor $\beta$-agonist, which is currently in a phase IIa clinical trial with NASH stage 1-3. After 12 weeks of administration, MGL-3196 was shown to reduce liver steatosis by $36 \%-42 \%$ as assessed by MRI-PDFF. Additionally, it had favorable effects on ALT and lipid parameters. ${ }^{56}$

Saroglitazar, MSDC-0602K, and IVA-337 are insulin sensitizers currently in phase IIA clinical trials. Saroglitazar is a dual PPAR- $\alpha / \gamma$ agonist that has been shown to reduce TGs, increase HDL, and improve glycemic control without the weight gain associated with PPAR- $\gamma$ agonists. ${ }^{57} \mathrm{~A}$ phase IIA clinical trial is underway for histologic endpoint assessment 
in NASH patients. MSDC-0602K is a mitochondrial target of thiazolidinediones, which produces several of the benefits of PPAR- $\gamma$ agonists with potentially less impact on weight gain and more impact on attenuating fibrosis. ${ }^{58}$ It is currently in phase IIa trial. ${ }^{34,59}$ IVA-337 (lanifibranor) is a pan-PPAR agonist $(\alpha / \delta / \gamma)$ that has been shown to reduce steatosis, reduce inflammation, reduce ballooning, improve insulin sensitivity, and reduce fibrosis in models of NASH. ${ }^{60}$ The NATIVE trial (NASH Trial to Validate IVA337 Efficacy) is a phase IIa clinical trial evaluating drug effects on histology in NASH patients. ${ }^{61}$

Volixibat is an apical sodium-dependent bile acid transporter inhibitor, which blocks the bile acid reabsorption, resulting in increased hepatic bile acid production, decreased cholesterol, and improved insulin sensitivity. It is currently in phase IIa trial with enrolling NASH subjects. The study endpoint is improvement in NAS without worsening fibrosis. ${ }^{34}$

Aramchol is a novel synthetic lipid molecule, which is obtained by conjugating cholic acid (bile acid) and arachidic acid (fatty acid) through an amide bond. Aramchol inhibits the stearoyl-coenzyme A desaturase 1 activity, which decreases lipogenesis and increases $\beta$-oxidation of fatty acids. In a phase IIb randomized, double-blind, placebo-controlled trial of 60 subjects with biopsy-confirmed NASH, Aramchol was given (100 mg or $300 \mathrm{mg}$ ) or placebo once daily for 12 weeks. The primary endpoint was the change in liver fat content on nuclear MR spectroscopy. There was a relative improvement in liver fat content in 12 weeks compared with placebo group. ${ }^{62}$ These findings are being further evaluated in phase IIb trial. ${ }^{62,63}$

Emricasan is a pan-caspase inhibitor, which blocks apoptotic and inflammatory caspase activation involved in hepatocyte death and has been shown to result in decreasing portal pressure. Subjects treated with emricasan over 28 days had $17.2 \%$ decrease in hepatic portal venous gas (HPVG) from baseline of $12 \mathrm{mmHg}$. There was also improvement in hepatic function tests. ENCORE-NF, a multicenter, randomized, double-blind, placebo-controlled phase IIb trial of emricasan in patients with NASH fibrosis, is underway. Subjects in the treatment arm were administered $5 \mathrm{mg}$ or 50 mg emricasan vs placebo for 72 weeks. The primary outcome is fibrosis improvement by one stage without worsening of steatohepatitis, improvement in NAS, and improvement in biomarker caspase 3/7.64 Another placebo-controlled, multicenter, double-blind, randomized phase II trial of emricasan demonstrated its safety in advanced liver disease, however, failed to prove the support of caspase inhibition for acute on chronic liver failure treatment. ${ }^{65,66}$

\section{Conclusion}

Due to the increase in global prevalence of NAFLD/NASH and lack of approved pharmacological agents, there is a surge for emerging clinical trials with agents targeting via different pathways. Data from these studies have shown promising results for reduction in steatosis, inflammation, and fibrosis. Several agents are being investigated in phase II and III clinical trials and are showing encouraging results. The next several years will likely see a therapeutic breakthrough in the approval of a pharmacologic agent for NASH; however, we must continue to emphasize the benefit of weight loss and increased activity as treatment modalities that remain cheap, effective, and with very few side effects.

\section{Disclosure}

The authors report no conflicts of interest in this work.

\section{References}

1. Onyekwere CA, Ogbera AO, Samaila AA, Balogun BO, Abdulkareem FB. Nonalcoholic fatty liver disease: Synopsis of current developments. Niger J Clin Pract. 2015;18(6):703-712.

2. Neuschwander-Tetri BA. Non-alcoholic fatty liver disease. BMC Med. 2017;15(1):45.

3. Chalasani N, Younossi Z, Lavine JE, et al. The diagnosis and management of nonalcoholic fatty liver disease: Practice guidance from the American Association for the Study of Liver Diseases. Hepatology. 2018;67(1):328-357.

4. Kleiner DE, Brunt EM, van Natta M, et al. Design and validation of a histological scoring system for nonalcoholic fatty liver disease. Hepatology. 2005;41(6):1313-1321.

5. Angulo P, Kleiner DE, Dam-Larsen S, et al. Liver fibrosis, but no other histologic features, is associated with long-term outcomes of patients with nonalcoholic fatty liver disease. Gastroenterology. 2015;149(2):389-397.

6. Ekstedt M, Hagström H, Nasr P, et al. Fibrosis stage is the strongest predictor for disease-specific mortality in NAFLD after up to 33 years of follow-up. Hepatology. 2015;61(5):1547-1554.

7. Musso G, Cassader M, Rosina F, Gambino R. Impact of current treatments on liver disease, glucose metabolism and cardiovascular risk in non-alcoholic fatty liver disease (NAFLD): a systematic review and meta-analysis of randomised trials. Diabetologia. 2012;55(4): 885-904.

8. Vilar-Gomez E, Martinez-Perez Y, Calzadilla-Bertot L, et al. Weight loss through lifestyle modification significantly reduces features of nonalcoholic steatohepatitis. Gastroenterology. 2015;149(2):367-378.

9. Sofi F, Macchi C, Abbate R, Gensini GF, Casini A. Mediterranean diet and health. Biofactors. 2013;39(4):335-342.

10. Ryan MC, Itsiopoulos C, Thodis T, et al. The Mediterranean diet improves hepatic steatosis and insulin sensitivity in individuals with non-alcoholic fatty liver disease. J Hepatol. 2013;59(1):138-143.

11. Kontogianni MD, Tileli N, Margariti A, et al. Adherence to the Mediterranean diet is associated with the severity of non-alcoholic fatty liver disease. Clin Nutr. 2014;33(4):678-683.

12. Markova M, Pivovarova $\mathrm{O}$, Hornemann S, et al. Isocaloric diets high in animal or plant protein reduce liver fat and inflammation in individuals with type 2 diabetes. Gastroenterology. 2017;152(3):571-585.

13. St George A, Bauman A, Johnston A, Farrell G, Chey T, George J. Independent effects of physical activity in patients with nonalcoholic fatty liver disease. Hepatology. 2009;50(1):68-76. 
14. Sanyal AJ, Chalasani N, Kowdley KV, et al. Pioglitazone, vitamin E, or placebo for nonalcoholic steatohepatitis. $N$ Engl J Med. 2010;362(18):1675-1685.

15. Lavine JE, Schwimmer JB, van Natta ML, et al. Effect of vitamin E or metformin for treatment of nonalcoholic fatty liver disease in children and adolescents: the TONIC randomized controlled trial. JAMA. 2011;305(16):1659-1668.

16. Miller ER 3rd, Pastor-Barriuso R, Dalal D, Riemersma RA, Appel LJ, Guallar E. Meta-analysis: high-dosage vitamin E supplementation may increase all-cause mortality. Ann Intern Med. 2005;142(1):37-46.

17. Abner EL, Schmitt FA, Mendiondo MS, Marcum JL, Kryscio RJ. Vitamin E and all-cause mortality: a meta-analysis. Curr Aging Sci. 2011;4(2):158-170.

18. Klein EA, Thompson IM, Tangen CM, et al. Vitamin E and the risk of prostate cancer: the Selenium and Vitamin E Cancer Prevention Trial (SELECT). JAMA. 2011;306(14):1549-1556.

19. Musso G, Cassader M, Paschetta E, Gambino R. Thiazolidinediones and advanced liver fibrosis in nonalcoholic steatohepatitis: a meta-analysis. JAMA Intern Med. 2017;177(5):633-640.

20. Yau H, Rivera K, Lomonaco R, Cusi K. The future of thiazolidinedione therapy in the management of type 2 diabetes mellitus. Curr Diab Rep. 2013;13(3):329-341.

21. Li Y, Liu L, Wang B, Wang J, Chen D. Metformin in non-alcoholic fatty liver disease: A systematic review and meta-analysis. Biomed Rep 2013;1(1):57-64.

22. Musso G, Gambino R, Cassader M, Pagano G. A meta-analysis of randomized trials for the treatment of nonalcoholic fatty liver disease. Hepatology. 2010;52(1):79-104.

23. Corey KE, Misdraji J, Gelrud L, Zheng H, Chung RT, Krauss RM. Nonalcoholic steatohepatitis is associated with an atherogenic lipoprotein subfraction profile. Lipids Health Dis. 2014;13(1):100.

24. Athyros VG, Tziomalos K, Gossios TD, et al. Safety and efficacy of long-term statin treatment for cardiovascular events in patients with coronary heart disease and abnormal liver tests in the Greek Atorvastatin and Coronary Heart Disease Evaluation (GREACE) Study: a post-hoc analysis. Lancet. 2010;376(9756):1916-1922.

25. Tikkanen MJ, Fayyad R, Faergeman O, et al. Effect of intensive lipid lowering with atorvastatin on cardiovascular outcomes in coronary heart disease patients with mild-to-moderate baseline elevations in alanine aminotransferase levels. Int J Cardiol. 2013;168(4):3846-3852.

26. Lewis JH, Mortensen ME, Zweig S, et al. Efficacy and safety of high-dose pravastatin in hypercholesterolemic patients with wellcompensated chronic liver disease: Results of a prospective, randomized, double-blind, placebo-controlled, multicenter trial. Hepatology. 2007;46(5):1453-1463.

27. del Ben M, Baratta F, Polimeni L, et al. Under-prescription of statins in patients with non-alcoholic fatty liver disease. Nutr Metab Cardiovasc Dis. 2017;27(2):161-167.

28. Konerman MA, Jones JC, Harrison SA. Pharmacotherapy for NASH: Current and emerging. J Hepatol. 2018;68(2):362-375.

29. Caldwell S, Ikura Y, Dias D, et al. Hepatocellular ballooning in NASH. J Hepatol. 2010;53(4):719-723.

30. Pavlides M, Banerjee R, Tunnicliffe EM, et al. Multiparametric magnetic resonance imaging for the assessment of non-alcoholic fatty liver disease severity. Liver Int. 2017;37(7):1065-1073.

31. Park CC, Nguyen P, Hernandez C, et al. Magnetic resonance elastography vs transient elastography in detection of fibrosis and noninvasive measurement of steatosis in patients with biopsy-proven nonalcoholic fatty liver disease. Gastroenterology. 2017;152(3):598-607.e2.

32. Sasso M, Audière S, Kemgang A, et al. Liver steatosis assessed by controlled attenuation parameter (CAP) measured with the XL probe of the FibroScan: a pilot study assessing diagnostic accuracy. Ultrasound Med Biol. 2016;42(1):92-103.

33. Dulai PS, Sirlin CB, Loomba R. MRI and MRE for non-invasive quantitative assessment of hepatic steatosis and fibrosis in NAFLD and NASH: Clinical trials to clinical practice. J Hepatol. 2016;65(5):1006-1016.
34. Golabi P, Bush H, Younossi ZM. Treatment strategies for nonalcoholic fatty liver disease and nonalcoholic steatohepatitis. Clin Liver Dis. 2017;21(4):739-753.

35. Fuchs M. Non-alcoholic fatty liver disease: the bile acid-activated farnesoid $\mathrm{x}$ receptor as an emerging treatment target. J Lipids. 2012;2012: $1-8$.

36. Ali AH, Carey EJ, Lindor KD. Recent advances in the development of farnesoid X receptor agonists. Ann Transl Med. 2015;3(1):5.

37. Neuschwander-Tetri BA, Loomba R, Sanyal AJ, et al. Farnesoid X nuclear receptor ligand obeticholic acid for non-cirrhotic, non-alcoholic steatohepatitis (FLINT): a multicentre, randomised, placebo-controlled trial. Lancet. 2015;385(9972):956-965.

38. Gawrieh S, Chalasani N. Emerging treatments for nonalcoholic fatty liver disease and nonalcoholic steatohepatitis. Clin Liver Dis. 2018;22(1):189-199.

39. Ratziu V, Sanyal AJ, Macconell L, et al. Regenerate: a phase 3, double-blind, randomized, placebo-controlled multicenter study of obeticholic acid therapy for nonalcoholic steatohepatitis. $J$ Hepatol. 2016;64(2):S294-S295.

40. la Cour Poulsen L, Siersbæk M, Mandrup S, editors. PPARs: Fatty Acid Sensors Controlling Metabolism. Seminars in Cell \& Developmental Biology. Elsevier; 2012;23(6):631-639.

41. Risérus U, Sprecher D, Johnson T, et al. Activation of peroxisome proliferator-activated receptor (PPAR)delta promotes reversal of multiple metabolic abnormalities, reduces oxidative stress, and increases fatty acid oxidation in moderately obese men. Diabetes. 2008;57(2):332-339.

42. Ratziu V, Harrison SA, Francque S, et al. Elafibranor, an agonist of the peroxisome proliferator-activated receptor- $\alpha$ and $-\delta$, induces resolution of nonalcoholic steatohepatitis without fibrosis worsening. Gastroenterology. 2016;150(5):1147-1159.

43. Lefebvre E, Moyle G, Reshef R, et al. Antifibrotic effects of the dual CCR2/CCR5 antagonist cenicriviroc in animal models of liver and kidney fibrosis. PLoS One. 2016;11(6):e0158156.

44. Miura K, Yang L, van Rooijen N, Ohnishi H, Seki E. Hepatic recruitment of macrophages promotes nonalcoholic steatohepatitis through CCR2. Am J Physiol Gastrointest Liver Physiol. 2012;302(11):G1310-G1321.

45. Friedman SL, Ratziu V, Harrison SA, et al. A randomized, placebocontrolled trial of cenicriviroc for treatment of nonalcoholic steatohepatitis with fibrosis. Hepatology. 2018;67(5);1754-1767.

46. Loomba R, Lawitz E, Mantry PS, Jaya-kumar S, Caldwell SH, Arnold H, editors. GS-4997, An Inhibitor of Apoptosis Signal-Regulating Kinase (ASK1), Alone or in Combination With Simtuzumab for the Treatment of Nonalcoholic Steatohepatitis (NASH): A Randomized, Phase 2 Trial. Hoboken (NJ): Wiley-Blackwell; 2016.

47. Clinical Trials.gov. Safety and Efficacy of Selonsertib in Adults With Nonalcoholic Steatohepatitis (NASH) and Bridging (F3) Fibrosis (STELLAR3). Available from: https://clinicaltrials.gov/ct2/show/ NCT03053050. Accessed August 3, 2018.

48. Clinical Trials.gov. Safety and Efficacy of Selonsertib in Adults With Compensated Cirrhosis Due to Nonalcoholic Steatohepatitis (NASH) (STELLAR 4). Available from: https://clinicaltrials.gov/ct2/show/ NCT03053063. Accessed August 3, 2018.

49. Malhi H, Camilleri M. Modulating bile acid pathways and TGR5 receptors for treating liver and GI diseases. Curr Opin Pharmacol. 2017;37:80-86.

50. Harrison SA, Abdelmalek MF, Trotter JF, et al. NGM282, a novel variant of FGF19, significantly reduces hepatic steatosis and key biomarkers of NASH: results of a phase 2, multicenter, randomized, double-blinded, placebo controlled trial in biopsy-confirmed NASH patients. J Hepatol. 2017;66(1):S92-S93.

51. Sanyal A, Charles ED, Neuschwander-Tetri B, et al. BMS-986036 (pegylated FGF21) in patients with non-alcoholic steatohepatitis: a phase 2 study. J Hepatol. 2017;66(1):S89-S90.

52. Marso SP, Daniels GH, Brown-Frandsen K, et al. Liraglutide and cardiovascular outcomes in type 2 diabetes. $N$ Engl J Med. 2016;375(4): $311-322$. 
53. Armstrong MJ, Gaunt P, Aithal GP, et al. Liraglutide safety and efficacy in patients with non-alcoholic steatohepatitis (LEAN): a multicentre, double-blind, randomised, placebo-controlled phase 2 study. Lancet. 2016;387(10019):679-690.

54. Sinha RA, Singh BK, Yen PM. Direct effects of thyroid hormones on hepatic lipid metabolism. Nat Rev Endocrinol. 2018;14(5):259-269.

55. Cable EE, Finn PD, Stebbins JW, et al. Reduction of hepatic steatosis in rats and mice after treatment with a liver-targeted thyroid hormone receptor agonist. Hepatology. 2009;49(2):407-417.

56. Harrison S. GS2 MGL-3196, a selective thyroid hormone receptor-beta agonist significantly decreases hepatic fat in NASH patients at 12 weeks, the primary endpoint in a 36 week serial liver biopsy study. Available from: http:/www.madrigalpharma.com/wp-content/uploads/2018/01/ Madrigal-Announces-EASL-Presentation.20180130_FINAL.pdf. Accessed August 3, 2018.

57. Sosale A, Saboo B, Sosale B. Saroglitazar for the treatment of hypertriglyceridemia in patients with type 2 diabetes: current evidence. Diab Metab Syndrome Obes. 2015;8:189-196.

58. Colca JR, Mcdonald WG, Mccommis KS, Finck BN. Treating fatty liver disease by modulating mitochondrial pyruvate metabolism. Hepatol Commun. 2017;1(3):193-197.

59. Fukunaga T, Zou W, Rohatgi N, Colca JR, Teitelbaum SL. An insulinsensitizing thiazolidinedione, which minimally activates PPAR $\gamma$, does not cause bone loss. J Bone Miner Res. 2015;30(3):481-488.

60. Wettstein G, Luccarini J.-M, Poekes L, Faye P, Kupkowski F, Adarbes $\mathrm{V}$. The new-generation pan-peroxisome proliferator-activated receptor agonist IVA337 protects the liver from metabolic disorders and fibrosis. Hepatol Commun [Internet]. 2017;1(6):524-537. Available from: http:// europepmc.org/abstract/MED/29404476. Accessed August 3, 2018.
61. Clinical Trials.gov. A Randomized, Double-blind, Placebo-controlled, Multicenter, Dose-range, Proof-of-concept, 24-week Treatment Study of IVA337 in Adult Subjects With Nonalcoholic Steatohepatitis (NASH). Available from: https://clinicaltrials.gov/ct2/show/NCT03008070. Accessed August 3, 2018.

62. Safadi R, Konikoff FM, Mahamid M, et al. The fatty acid-bile acid conjugate Aramchol reduces liver fat content in patients with nonalcoholic fatty liver disease. Clin Gastroenterol Hepatol. 2014;12(12): 2085-2091.

63. Clinical Trials.gov. A Clinical Trial to Evaluate the Efficacy and Safety of Two Aramchol Doses Versus Placebo in Patients With NASH (Aramchol_005). Available from: https://clinicaltrials.gov/ct2/show/ NCT02279524. Accessed August 3, 2018.

64. Clinical Trials.gov. Emricasan, a Caspase Inhibitor, for Evaluation in Subjects With Non-Alcoholic Steatohepatitis (NASH) Fibrosis (ENCORE-NF). Available from: https://clinicaltrials.gov/ct2/show/ NCT02686762. Accessed August 3, 2018.

65. Clinical Trials.gov. Emricasan, a Caspase Inhibitor, for Treatment of Subjects With Decompensated NASH Cirrhosis (ENCORE-LF). Available from: https://clinicaltrials.gov/ct2/show/NCT03205345. Accessed August 3, 2018.

66. Ir.conatuspharma.com. Conatus Announces Exercise of License Option for Global Development and Commercialization of Emricasan Following Notice of Initiation of Phase 2b ENCORE-LF Clinical Trial in NASH Cirrhosis (NASDAQ:CNAT). Available from: http:// ir.conatuspharma.com/releasedetail.cfm?releaseid=1024672. Accessed August 3, 2018.
Diabetes, Metabolic Syndrome and Obesity: Targets and Therapy is an international, peer-reviewed open-access journal committed to the rapid publication of the latest laboratory and clinical findings in the fields of diabetes, metabolic syndrome and obesity research. Original research, review, case reports, hypothesis formation, expert opinion and commentaries are all considered for publication. The manuscript management system is completely online and includes a very quick and fair peer-review system, which is all easy to use. Visit http://www.dovepress.com/testimonials.php to read real quotes from published authors. 